\title{
Optimal Rational Function Approximation for Fractional Integral and Differential Operators
}

\author{
Zhang Xuxiu,Yao Xin \\ School of Electronics and Information Engineering \\ Dalian Jiaotong University \\ Dalian, China \\ zhangxuxiu@163.com
}

\begin{abstract}
Bode diagram based rational function approximation method for fractional integral and differential operators are analyzed in detail. For the approximation rational function orders is the lowest under satisfying approximation accuracy in the approximation frequency interval, two steps are proposed: 1) Choose reasonable initial and terminal frequency of rational function logarithmic amplitude-frequency characteristic. 2) Set approximation error of logarithmic amplitude-frequency characteristic by taking the error between asymptote and exact value into account. Computation examples demonstrate the validity of this method.
\end{abstract}

Key words-Fractional integral and differential operators; Rational function approximation; Bode diagram; Optimal rational function approximation

\section{INTRODUCTION}

Fractional Order Calculus (FOC) was first proposed in 1695[1]. However, due to a lack of knowledge of its physical significance and geometric meaning, FOC has laid dormant for a long time. It's not until recent decades, as with the development of computer science and its application, FOC has gained much attention in scientific and engineering community[2]. FOC has broad application in complex systems modeling, analysis and identification, signal processing and automatic control[2] and so on. FOC can resolve some problems that traditional integer order calculus could not do. Some previously undiscovered or unexplained phenomenon can be discovered or explained by FOC. For example, the conventional automatic control system, its performance would be further improved by FOC modeling or FOC controller[4].

Fractional order integral or differential operator $1 / s^{\gamma}$ $(0<\gamma<1$ and $-1<\gamma<0$ are integral and differential respectively) is the most common fractional filter in automatic control system. It should be discretized by an approximate model in digital control systems. Rational function of Laplace operator $s$ is used to approximate $1 / s^{\gamma}$ in continuous control systems. This paper is aimed at the rational function approximation for $1 / s^{\gamma}$.Existing methods[5-9] include continuous fractional expansion (CFE),

\author{
Fei Jiyou \\ School of Bullet Train Application and Maintenance \\ Engineering \\ Dalian Jiaotong University \\ Dalian, China \\ YAOXIN52818989@126.com
}

Carlson, Matsuda,Oustloup(CRONE),etc. A common feature of these methods is that they do not take how to obtain the rational function of lowest order under specified conditions (approximation frequency range and approximation error) into account, namely how to achieve the best rational function approximation. This paper proposes an optimal rational function approximation method for $1 / s^{\gamma}$.

This paper is organized as follows: Section 2 deals with the proposal of rational function approximation based on Bode diagram for fractional integral operator. Section 3 describes the method of setting approximation error bandwidth. Section 4 gives out the solving issue and improving it. And section 5 is the conclusion part.

\section{BODE DIAGRAM BASED RATIONAL FUNCTION} APPROXIMATION FOR FRACTIONAL INTEGRAL OPERATOR

\section{A. Mathematical model}

Fractional integral operator is $1 / s^{\gamma}$, where $0<\gamma<1$. Assume it is in series with proportional coefficient $k_{0}$.The transfer function is $G_{0}(s)=k_{0} / s^{r}$. It can be approximated by $R(s)$, where $R(s)$ is a rational function of $s$. And

$$
R(s)=K \frac{\left(s+b_{1}\right)\left(s+b_{2}\right) \ldots}{\left(s+a_{1}\right)\left(s+a_{2}\right)\left(s+a_{3}\right) \ldots}
$$

Where $K$ is an undetermined proportion coefficient. $a_{i}$ and $b_{i}$ are undetermined constants. The frequency characteristic is $G_{0}(j \omega)=k_{0} /(j \omega)^{r}$.

$$
R(j \omega)=K \frac{\left(s+b_{1}\right)\left(s+b_{2}\right) \ldots}{\left.\left(s+a_{1}\right)\left(s+a_{2}\right)\left(s+a_{3}\right) \ldots\right|_{s=j \omega}}
$$

Thus the problem of $R(s)$ approximating $G_{0}(s)$ in $s$ domain is transferred to $R(j \omega)$ approximating $G(j \omega)$ in the frequency domain. $R(s)$ approximating to $G_{0}(s)$ by Bode diagram of logarithmic amplitude-frequency characteristics (LAFC) in the paper. 


\section{B. Amplitude frequency characteristics}

Fig.1 shows LAFCs of $G_{0}(s)$ and its approximation rational function $R(s)$, where LAFC is a line $L_{0}$. Lines $L_{1}$ and $L_{2}$ are parallel with $L_{0}$. The LAFC of $R(s)$ is composed of polyline inside $L_{1}$ and $L_{2}$. The polyline is connected by horizontal lines and oblique lines with slope $-20 d B / d e c$ alternately. Maximum longitudinal distance between polyline and $L_{0}$ is maximum error (absolute value) $\delta$. The area inside $L_{1}$ and $L_{2}$ is error band. The frequency corresponding to the intersection of the polyline with $L_{1}$ or $L_{2}$ is called handover frequency. From left to right, handover frequencies are divided into two groups: one group corresponding to the initial of horizontal segment, called as $b_{i}$, another is to the initial of oblique segment, denoted by $a_{i}$. Two special handover frequencies corresponding to initial and terminal of polyline, are denoted by $\Omega_{1}$ and $\Omega_{2}$ respectively. $\left[\omega_{a}, \omega_{b}\right]$ is approximation frequency range. $\left[\Omega_{1}, \Omega_{2}\right]$ is called rational function build frequency range. $\left[\omega_{a}, \omega_{b}\right] \in\left[\Omega_{1}, \Omega_{2}\right]$. At two small ranges by the right of $\Omega_{1}$ and left of $\Omega_{2}$, LAFC of $R(s)$ is within error band, its phase-frequency characteristic approximation error is larger than normal. In order to exclude these two small ranges from the approximate frequency range, the approximation frequency range should be less than the build frequency range. As shown in Fig.1.

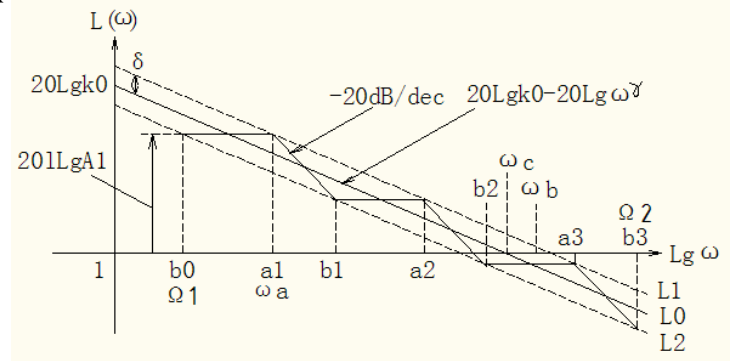

Figure1. LAFCs of $G_{0}(s)=k_{0} / s^{\gamma}, R(s), \Omega_{2}=b_{j}, j=3$

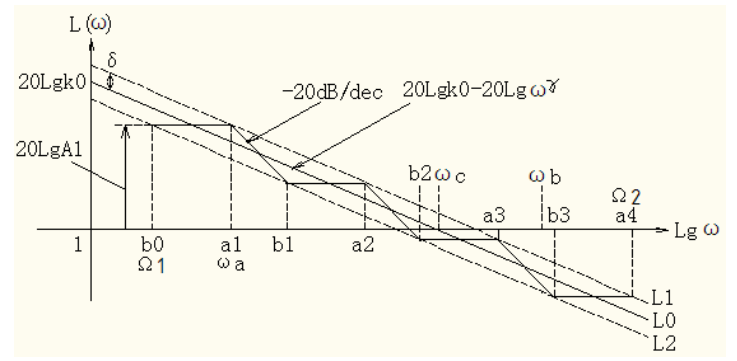

Figure2. LAFCs of $G_{0}(s)=k_{0} / s^{\gamma} R(s), \Omega_{2}=a_{j}, j=4$

\section{Calculation of the handover frequency}

After $\omega_{a}$ and $\omega_{b}$ have been set, handover frequencies is $a_{1}=\omega_{a}$. In Fig.1, we have

$$
\begin{aligned}
& 20 \lg k_{0}-20 \lg a_{1}^{\gamma}+\delta=20 \lg k_{0}-20 \lg a_{1} \\
& 20 \lg k_{0}-20 \lg b_{1}^{\gamma}-\delta=20 \lg k_{0}-20 \lg b_{1}
\end{aligned}
$$

Subtract two equations, we get the solution $b_{1}=a_{1} 10^{\frac{\delta}{10(1-\gamma)}}$.

Again, $20 \lg k_{0}-20 \lg b_{1}^{\gamma}-\delta=20 \lg k_{0}-20 \lg a_{2}^{\gamma}+\delta$.

Predigest it as $20 \lg \left(a_{2} / b_{1}\right)^{\gamma}=2 \delta$, Solution is $a_{2}=b_{1} 10^{\frac{\delta}{10 \gamma}}$.Similarly, we yield the recurrence formulas

$$
\begin{aligned}
& b_{i}=a_{i} 10^{\frac{\delta}{10(1-\gamma)}}, \quad i=1,2,3, \ldots \\
& a_{i+1}=b_{i} 10^{\frac{\delta}{10 \gamma}}, \quad i=1,2,3, \ldots
\end{aligned}
$$

Specifically, the adjacent handover frequency ratios are $b_{i} / a_{i}=10$, when $\gamma=5, \delta=5$, respectively.

D. Calculation of Polyline initial and terminal frequencies

Polyline initial frequency is obtained by when $i=0, a_{1}=b_{0} 10^{\frac{\delta}{10 \gamma}}$,

$$
\Omega_{1}=b_{0}=a_{1} 10^{\frac{-\delta}{10 \gamma}}
$$

The polyline terminal frequency is based on comparing values of $a_{i+1}$ (or $b_{i}$ ) with $\omega_{b}$. The steps are as follows:

1) In the case of $i+1=j$, obtained $a_{j}$.If $a_{j} \geq \omega_{b}$, then

$$
\Omega_{2}=b_{j}
$$

2) In the case of $i=j$, obtained $b_{j}$. If $b_{j} \geq \omega_{b}$, then

$$
\Omega_{2}=a_{j+1}
$$

\section{E. Generation of approximation rational function}

Approximation rational function can be obtained after calculating handover frequencies based on above algorithm. Each $a_{i}$ is corresponding to an inertial element, which is a factor in denominator of $R(s)$. Similarly, every $b_{i}$ is to a first-order differential element in numerator of $R(s)$ except polyline initial and terminal frequency.

Transfer function of first inertial element is denoted as

$$
G_{1}(s)=\frac{A_{1}}{1+s / a_{1}}
$$

As $20 \lg A_{1}=20 \lg k_{0}-20 \lg a_{1}^{\gamma}+\delta, \quad k_{0}=\omega_{c}^{\gamma}$, then 


$$
A_{1}=\left(\frac{\omega_{c}}{a_{1}}\right)^{\gamma} 10^{\frac{\delta}{20}}
$$

Similarly, $b_{i}$ and $a_{i}$ are differential and inertial element alternately, their coefficients are reciprocal and can be reduced in series product. Finally, for Fig.1 we have

$$
R(s)=10^{\frac{\delta}{20}}\left(\frac{\omega_{c}}{a_{1}}\right)^{\gamma} \frac{\left(1+s / b_{1}\right)\left(1+s / b_{2}\right)}{\left(1+s / a_{1}\right)\left(1+s / a_{2}\right)\left(1+s / a_{3}\right)} .
$$

Fig. 2 shows

$$
R(s)=10^{\frac{\delta}{20}}\left(\frac{\omega_{c}}{a_{1}}\right)^{\gamma} \frac{\left(1+s / b_{1}\right)\left(1+s / b_{2}\right)\left(1+s / b_{3}\right)}{\left(1+s / a_{1}\right)\left(1+s / a_{2}\right)\left(1+s / a_{3}\right)} .
$$

The general forms of above two expression are as follows:

$$
\begin{aligned}
& R(s)=10^{\frac{\delta}{20}}\left(\frac{\omega_{c}}{a_{1}}\right)^{\gamma} \frac{\left(1+s / b_{1}\right)\left(1+s / b_{2}\right) \ldots\left(1+s / b_{j-1}\right)}{\left(1+s / a_{1}\right)\left(1+s / a_{2}\right) \ldots\left(1+s / a_{j}\right)} \\
& R(s)=10^{\frac{\delta}{20}}\left(\frac{\omega_{c}}{a_{1}}\right)^{\gamma} \frac{\left(1+s / b_{1}\right)\left(1+s / b_{2}\right) \ldots\left(1+s / b_{j}\right)}{\left(1+s / a_{1}\right)\left(1+s / a_{2}\right) \ldots\left(1+s / a_{j}\right)}
\end{aligned}
$$

\section{SETTING APPROXIMATION ERROR BANDWIDTH}

For setting approximation frequency range $\left[\omega_{a}, \omega_{b}\right]$, we note that the wider approximation error bandwidth is, the less the number of polyline inflection point and handover frequency are, and the lower order of approximation function is. In order to reduce the orders and optimize rational approximation function, we should increase the approximation error bandwidth to the best of one's abilities within acceptable approximation error. Therefore, two problems should be considered:1)The error between the asymptote and exact value of LAFC.2) The relationship between pole frequency of LAFC approximation error curve and adjacent handover frequency.

\section{A. The error between the asymptote and exact value of $L A F C$}

LAFC of approximation rational function are represented by asymptote in Bode diagram. There is error between asymptote and accurate characteristics. The closer to the handover frequency, the greater the error is. Maximum error is on the handover frequency. For inertia element, the maximum error is $3 d B$. For first-order differential element, the maximum error is $-3 d B$. The asymptote is always outside of the accurate characteristics. If error bandwidth in Bode diagram is set directly according to the LAFC maximum acceptable approximation error, then it will result in higher order of $R(s)$. And the actual approximation error is less than the setting value, which means the approximation precision has margin. Eliminating this margin can reduce the orders of $R(s)$. Therefore, error band should be widened appropriately. Related problems are discussed as follows.

\section{$B$. The relationship between approximation error curve pole frequency and the LAFC handover frequency}

The LAFC expression of fractional integral operator $k_{0} / s^{\gamma}$ is $20 \lg k_{0}+20 \lg \omega^{-\gamma}$.

Assume $A_{j} /\left(1+s T_{j}\right)$ is a transfer function of certain inertial element in the approximation rational function, its LAFC expression is $20 \lg A_{j}-20 \lg \sqrt{1+\left(\omega T_{j}\right)^{2}}$. The first expression subtracts second one. Let derivative of the difference for $\omega$ is equal to zero, we obtain $\frac{d}{d \omega}\left[\lg \omega^{-\gamma}+\lg \sqrt{1+\left(\omega T_{j}\right)^{2}}\right]=0$.

Solution of this equation is namely a pole frequency of approximation error curve, denote by $\omega^{*}$. Then

$$
\omega^{*}=\frac{1}{T_{j}} \sqrt{\frac{\gamma}{1-\gamma}}=\omega_{j} \sqrt{\frac{\gamma}{1-\gamma}}
$$

where $\omega_{j}$ is a handover frequency.

\section{Setting approximation error bandwidth}

Assume the maximum acceptable error of amplitude is $\mu$. Convert $\mu$ to the error of LAFC in Bode diagram $\delta_{0}=20 \lg \mu$.The error bandwidth (single side) should be taken $\delta=\delta_{0}+\Delta \delta$.where $\delta$ is the difference between approximation function LAFC asymptote and LAFC of fractional integral operator at $\omega_{j}, \delta_{0}$ is the difference of approximation function LAFC exact value and fractional integral operator LAFC at $\omega^{*} . \Delta \delta$ is a correction value related to $\omega^{*}$ and $\omega_{j}$. There are three cases as follows.

(1) $\gamma=0.5$, as shown in (12) $\omega^{*}=\omega_{j}$, the error between the asymptote and the exact value of the approximation function LAFC at $\omega_{j}$ equals to $3 d B$. That is $\Delta \delta=\delta-\delta_{0}=3 d B$.

(2) $\gamma>0.5$, as (12), $\omega^{*}>\omega_{j}$, the error between of the exact value of the approximation function LAFC and fractional integral operator LAFC is $\delta_{0}$ at $\omega^{*}$ and is $\delta_{1}$ at $\omega_{j}$. Moreover, $\delta-\delta_{1}=3 d B$ and $\delta_{0}>\delta_{1}$. So we obtain $\Delta \delta=\delta-\delta_{0}<3 d B$.

(3) $\gamma<0.5$,as (12), $\omega^{*}<\omega_{j}$.Similarly get $\Delta \delta=\delta-\delta_{0}<3 d B$.

The farther $\gamma$ value deviates from 0.5 , the smaller $\Delta \delta$ is. There is no analytical methods to determine $\Delta \delta$ value, but it can be selected by the experience or testing. For instance, when $\gamma=0.4$ or $\gamma=0.6$, 
$\Delta \delta \approx 2.8 d B$. When $\gamma=0.2$ or $\gamma=0.8$, take $\Delta \delta \approx 2 d B$, and so on.

\section{IMPROVEMENT}

Let $G_{0}(s)=1 / s^{0.5}$. Find $R(s)$ to get the maximum approximation error of the LAFC (exact value) $\delta_{0}=2 d B$ in the frequency range $\left[\omega_{a}, \omega_{b}\right]=\left[1,10^{4}\right] \quad(\mathrm{rad} / \mathrm{s}) \quad$.we have $R(s)=10^{\frac{\delta}{20}}\left(\frac{\omega_{c}}{a_{1}}\right)^{\gamma} \frac{\left(1+s / b_{1}\right)\left(1+s / b_{2}\right)}{\left(1+s / a_{1}\right)\left(1+s / a_{2}\right)} \quad$.LAFC and phasefrequency characteristics curves are as follows.

Here since before decreasing $a_{1}$ selecting $\Omega_{2}=a_{3}$ is based on $b_{2}>\omega_{b}$ but not $b_{2}=\omega_{b}$, so that the decrease of $a_{1}$ cannot change $\Omega_{2}$ and orders of $R(s)$. Contrarily if it is based on $b_{2}=\omega_{b}$ (is in critical case) then decrease of $a_{1}$ will bring change of $\Omega_{2}$ and increase of orders of $R(s)$. Commonly if selecting $\Omega_{2}$ is based on equation of formula $a_{j} \geq \omega_{b} \quad\left(\right.$ or $\left.b_{j} \geq \omega_{b}\right)$ then decrease of $a_{1}$ will bring increase of $R(s)$ orders . Otherwise it is based on inequation then it is possible that the orders of $R(s)$ do not increase.
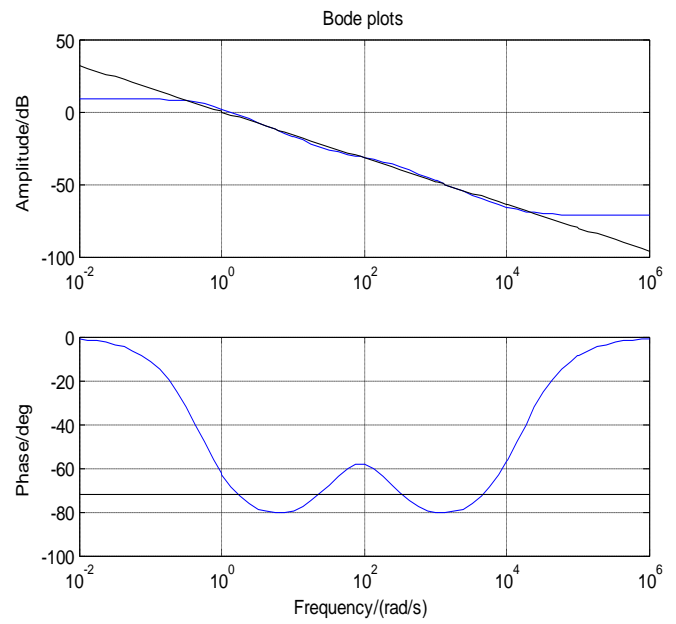

Figure3. LAFC and phase-frequency characteristics curves

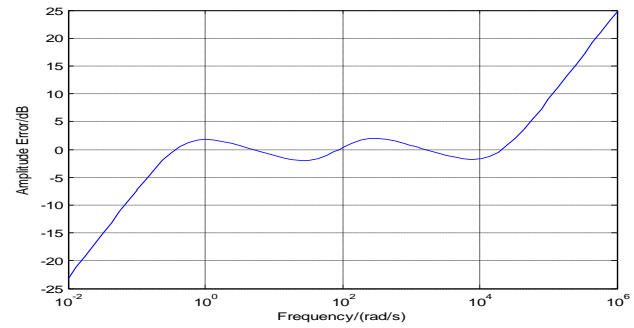

Figure4. Approximation error curve of LAFC
The approximation of fractional differential operator, $\left(1 / k_{0}\right) s^{\gamma}$,can utilize above optimal rational function approximation method of fractional integral operator. Here reverse numerator and denominator of $\left(1 / k_{0}\right) s^{\gamma}$,we get $k_{0} / s^{\gamma}$. Find the approximation rational function $R(s)$ of this $k_{0} / s^{\gamma}$.Furthermore, we reverse numerator and denominator of this $R(s)$ and change sign of order in MATLAB for drawing Bode diagram.

\section{CONCLUSIONS}

We have addressed fractional integral operator optimal rational function approximation method based on Bode diagram. The so-called optimal means: Under the condition of the approximate frequency range and the maximum acceptable amplitude approximation error (and corresponding to maximum phase error), the orders of the rational function is the lowest. The previous method can also be applied to fractional differential operator approximation. Calculation examples show availability of above works.

\section{ACKNOWLEDGMENT}

This work is supported by the National Science Foundation of China under the grant 61201419/F0103.

\section{REFERENCES}

[1] Donato Cafagna, Fractional Calculus: A Mathematical Tool from the Past for Present Engineers, IEEE Industrial Electronics magazine,2007,pp 35-40.

[2] J.A.Tenreiro Machado. Application of Fractional Calculus in Engineering Sciences,IEEE 6th International conference on computational cybernetics,Nov.27-29,2008,Stara Lesna,Slovakia,pp 11-14.

[3] Caibin Zeng, Qigui Yang, YangQuan Chen. Solving nonlinear stochastic differential equations with fractional Brownian motion using reducibility approach, Nonlinear Dynamics. 2012, 67(3):pp2719-2716.

[4] Kouyou Sawai, Takahiro Takamatisu,Hiromitsu Ohmori. Adaptive Control Law using Fractional Calculus Systems. SICE Annual Conference 2012. August 20-23, 2012, Akita University, Akita, Japan,pp1502-1505.

[5] YangQuan Chen,Blas M. Vinagre,Igor Podlubny, Using Continued Fraction Expansion to Discretize Fractional Order Derivatives, Nonlinear Dynamics, Vol. 38, Nos. 1-4, December 2004, pp 155-170.

[6] Li Wen, Zhao Hui-min. Rational Function Approximation for Fractional Order Differential and Integral Operators. Acata Automatica Sinica, 2011, 37(8), pp 999-1005

[7] Sehoon Oh, Yoichi Hori. Realization of Fractional Order Impedance by Feedback Control,The 33rd Annual Conference of the IEEE industrial Electronics Society,2007,pp299-304.

[8] Santouh Z, Charef A, Assabaa M. Approximation of multiple fractional order systems. Arab Research Institute in Sciences and Engineering, 2007, 3(4),pp 155-161.

[9] Zhao Hui-Min, Li Wen, Deng Wu. Approximation degree selection for one kind of fractional-order filter. Electric Machines and Control, 2010, 14(1),pp 90-94. 